This item was submitted to Loughborough's Research Repository by the author.

Items in Figshare are protected by copyright, with all rights reserved, unless otherwise indicated.

\title{
Multiphysics analysis for the determination of valvetrain characteristics
}

PLEASE CITE THE PUBLISHED VERSION

PUBLISHER

Professional Engineering Publishing / @ IMECHE

VERSION

VoR (Version of Record)

LICENCE

CC BY-NC-ND 4.0

REPOSITORY RECORD

Teodorescu, M., Vasilis Votsios, and Homer Rahnejat. 2019. "Multiphysics Analysis for the Determination of Valvetrain Characteristics". figshare. https://hdl.handle.net/2134/4828. 
This item was submitted to Loughborough's Institutional Repository (https://dspace.lboro.ac.uk/) by the author and is made available under the following Creative Commons Licence conditions.

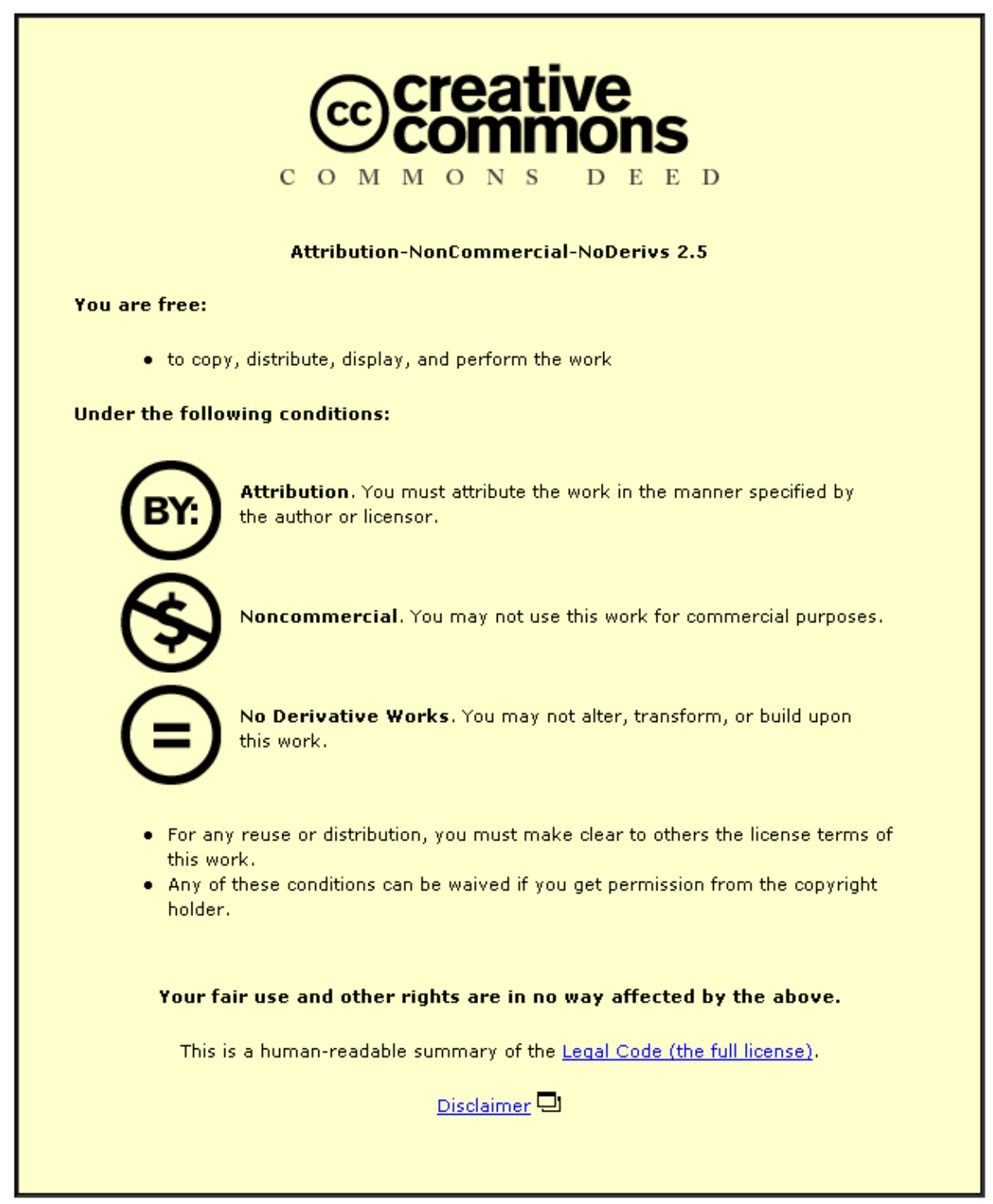

For the full text of this licence, please go to: http://creativecommons.org/licenses/by-nc-nd/2.5/ 


\title{
Multiphysics analysis for the determination of valvetrain characteristics
}

M Teodorescu ${ }^{\ddagger}$, V Votsios, and H Rahnejat*

Wolfson School of Mechanical and Manufacturing Engineering, University of Loughborough, Loughborough, UK

The manuscript was received on 29 October 2004 and was accepted after revision for publication on 4 May 2005.

DOI: 10.1243/095440705X34801

\begin{abstract}
The ideal function of a valvetrain system is to synchronize the opening and closing of the inlet and exhaust valves with the required thermodynamics of the combustion process. As such, ideally a kinematic-type mechanism is desired. However, the timing requirements in the action of each valve and between any inlet-exhaust pair necessitate the use of contacting pairs of suitable profiles. The very existence of contact renders the problem one of complex non-linear dynamics, which is further exacerbated by the translational imbalance of the reciprocating compliant elements such as the valve itself. The interplay between these various forms of dynamics, inertial, structural, and impact/contact, make the problem quite complex to analyse. As a result, some of the most important problems with valvetrains are only surmised at, rather than fundamentally understood. The multiphysics modelling approach proposed in this paper renders a better understanding, as well as conforming to experimental observations.
\end{abstract}

Keywords: valvetrain system, multiphysics analysis, impact dynamics

\section{INTRODUCTION}

The valvetrain is one of the most highly loaded vehicular powertrain subsystems, which contributes to a host of undesired phenomena. These include a sizeable contribution to both mechanical and frictional losses in a four-stroke IC engine. The former is due to high out-of-balance inertial forces generated by the translational motion of its components, such as the valve, the pushrod, or the valve spring of non-negligible mass. The latter is due to insufficient lubrication in a number of contact zones, chiefly between the cam and the follower. Valvetrain systems with single or double overhead cams, used in many modern vehicles, have reduced inertial and friction problems.

An inadequate film thickness may result, due to a number of reasons. One is the momentary cessation

\footnotetext{
* Corresponding author: Wolfson School of Mechanical and Manufacturing Engineering, University of Loughborough, Loughborough LE11 3TU, UK. email: r.rahnejat@lboro.ac.uk ${ }^{\ddagger}$ Currently at School of Engineering and Mathematical Sciences, City University, London, UK
}

of entraining motion, prior to and just past the cam nose-follower contact as the inlet reversal takes place [1]. At higher cam angular velocities, the lubricant film is replenished rapidly. However, at higher speeds of revolution, the geometrical acceleration due to cam lift can induce high enough inertial forces to result in valve spring surge, valve toss, and, consequently, loss of cam-follower contact, referred to as tappet jump $[2,3]$. Contact separation can result in loss of lubrication, and the subsequent bounce of the cam upon the tappet can introduce a significant impact force. Therefore, tribological study of the cam-tappet pair cannot be divorced from the dynamics of the valvetrain system as a whole.

At high inertial forces the structural response of the system components can play an important role, particularly in modern motor vehicles, where materials of lighter but durable construction are increasingly used in order to reduce the inertial imbalance, but at the same time withstand the generated forces. This, however, results in their structural deformation and vibration $[2,4]$. Therefore, for modern engines rigid-body dynamic analysis is not an appropriate approach. An elastodynamic analysis must be carried out. 
Most valvetrain analyses are confined to either the study of dynamics of the system or its tribological performance for a given contact zone. This approach is not holistic and ignores their interplay, which leads to tribo-elasto-multi-body dynamics. In short, a multiphysics analysis approach is required, encompassing large rigid-body displacements of rigid elements, small amplitude vibration of elastic members, and elastohydrodynamics of cam-follower contact conjunction in a single analysis framework. This approach is termed 'multiphysics'. It is shown that this approach yields predictions that conform much closer to experimental findings than the traditional rigid-body dynamics, even at modest camshaft speeds.

In most cases, by increasing the accuracy of a theoretical simulation, the amount of computer calculations increases exponentially. In the present work, lubrication and dynamic models were devised to describe the physical phenomena, starting from basic principles, with an acceptable analysis time.

To check the validity of the valvetrain dynamic model, the predictions have been compared with the measurements on a single-cylinder diesel engine.

In the analysis carried out here, the smallamplitude lateral and tilting motion of the valve is ignored, which is clearly present in the actual measurements. One repercussion of these secondary motions of the valve is tappet spin, which introduces complex problems of its own, as described by Teodorescu and Taraza [5].

\section{THEORETICAL FORMULATION}

The model includes the overall inertial dynamics of the valvetrain components, including the valve, the rocker arm, the push rod (incorporating the flat follower, referred to as the tappet), and the cam. The elastic behaviour of the various elements are incorporated, initially, by equivalent stiffness and damping in each case. Characteristics of contacting/ impacting pairs are also included in the formulation.

Figure 1(a) shows a typical valvetrain mechanism, and a two-mass dynamic model developed to represent it. The two inertial elements represent the mass of the valve and all its attachments (including the retainer and one third of the non-negligible mass of the valve spring) and that of the pushrod/flat follower and the proportion of the rocker arm in translation (Teodorescu [6]). The various stiffness and damping components in the model are described in the notation. The 'floor' movement in this model,

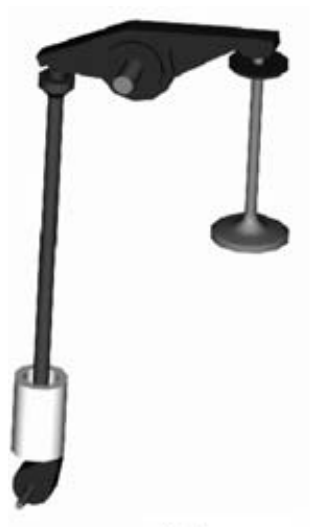

(a)

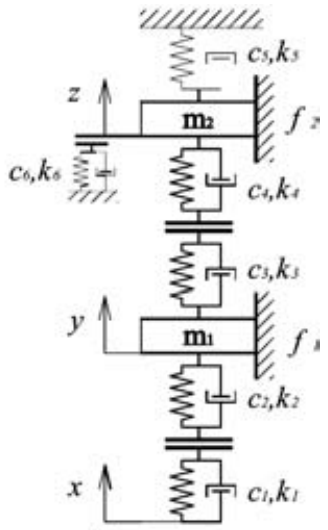

(b)
Fig. 1 Valvetrain model

denoted by $x$, represents the movement of the surface of the cam, which adheres to the flat follower surface by the contact compliance indicated by $k_{1}, c_{1}$. The motion of the cam is regarded as kinematic as there is no translational imbalance associated with it. The motion of the follower/pushrod assembly is denoted by $y$, whereas the movement of the valve itself is denoted by $z$.

\subsection{Inertial dynamics, incorporating system compliances}

In this model the element $c_{6}, k_{6}$ represents the valvevalve seat contact, which acts in parallel to that of the contact between the valve and the rocker arm as shown in Fig. 1(b) for this two-degrees-of-freedom system. The equations of motion are

$$
\begin{aligned}
& m_{i} \ddot{l}_{i}+c_{\mathrm{e}_{i}}\left(\dot{l}_{i}-\dot{l}_{i-1}\right)+k_{\mathrm{e}_{i}}\left(l_{i}-l_{i-1}\right)+c_{\mathrm{g}_{i}} \dot{l}_{i} \\
& \quad-k_{\mathrm{e}_{i+1}}\left(l_{i+1}-l_{i}\right)-c_{\mathrm{e}_{i+1}}\left(\dot{l}_{i+1}-\dot{l}_{i}\right)+F_{i}^{m}=0
\end{aligned}
$$

where

$$
k_{\mathrm{e}_{i}}=\frac{k_{i} k_{i+1}}{k_{i}+k_{i+1}}, \quad c_{\mathrm{e}_{i}}=\frac{c_{i} c_{i+1}}{c_{i}+c_{i+1}}, \quad i=1,2
$$

The kinematic equation of motion of the cam is described by the cam lift as the floor excitation in the model, shown in Fig. 1(b). To include this in a precise manner, measured valve acceleration is integrated twice. Since the valve acceleration was measured on a fired combustion engine, the signal contains, besides the geometrical acceleration, the mechanical vibrations of the mechanism. To minimize the extraneous influence of engine noise, the computed tappet lift was carefully filtered and a polynomial curve corresponding to an automotive cam lift was fitted. The geometrical tappet lift for this engine is symmetrical, and the general form for half of the event has been derived from Chen [7] and 
Teodorescu and Taraza [5] as

$$
x(t)=x_{0}\left(1+\sum_{i}^{k} C_{i}(\omega t)^{r_{i}}\right)+\varepsilon_{\text {res }}
$$

where

$$
C_{i}=\frac{\prod_{j=1, j \neq i}^{k} r_{j}}{\prod_{j=1, j \neq i}^{k}\left(r_{j}-r_{i}\right)}
$$

The residual error term is $\varepsilon_{\text {res }}$, between the modelled tappet lift and the measured one, which is quite small. The ideal polynomial tappet lift minimizes the error without excessively increasing the order of polynomial. The best agreement was obtained by a $26^{\text {th }}$ degree polynomial with the following parameters

$$
k=5, \quad r_{1}=2, \quad r_{2}=8, \quad r_{3}=14, \quad r_{4}=20, \quad r_{5}=26
$$

\subsection{Kinematic conditions in cam-tappet conjunction}

To determine the tribological conditions between the cam and the tappet, it is necessary to obtain the instantaneous speed of lubricant entrainment into the contact conjunction, which is the average speed of the two mating surfaces as described by Kushwaha and Rahnejat [2]

$$
u=\frac{u_{1}+u_{2}}{2}
$$

The surface velocity of the cam $u_{1}$ depends upon the effective instantaneous contact radius and the angular velocity of the cam as

$$
u_{1}=R_{\mathrm{c}} \times \omega
$$

This effectively considers the tappet to have no translational velocity with respect to the cam in the direction of entraining motion. However, the tappet spins, which has been investigated in detail by Teodorescu and Taraza [5], and is not taken into account in this paper. The effective contact radius is due to the cam lift and the geometrical acceleration, and it is obtained as

$$
R_{\mathrm{c}}=R_{\mathrm{b}}+x+\frac{\ddot{x}}{\omega^{2}}
$$

Thus, the speed of entraining motion is obtained as

$$
u=\left(R_{\mathrm{c}}+\frac{\ddot{x}}{\omega^{2}}\right) \frac{\omega}{2}
$$

To obtain an estimate of the lubricant film thickness during the cam cycle, the operating parameters of interest are: the speed of entraining motion, the contact/impact load and the contribution due to the squeeze-film action, the rheology of the lubricant, and the elastic properties of the contiguous solids in contact [1]. This can be obtained either by the simultaneous solution of the Reynolds equation, the elastic film shape, and the lubricant state equations in a transient manner [1], or by using an approximate, extrapolated oil-film thickness formula (which is usually obtained by a series of quasi-static approximations) [2]. The latter approach lends itself to an acceptable computation time solution, but leads to an underestimation of film thickness, and a corresponding overestimation of lubrication film reaction as highlighted by Jalali-Vahid et al. [8]. However, for most of the cam cycle a reasonable estimate of both these quantities is obtained, given that a suitable formula pertaining to the contact geometry is used, this being a finite line configuration. The only known formula for the central oil-film thickness for such a conjunction, incorporating the combined effect of lubricant entrainment and squeeze-film action, is given by Rahnejat [9] for the case of oil-film thickness in the centre of the contact footprint as

$$
h_{\mathrm{o}}^{*}=1.67 G^{* 0.421} U^{* 0.541} F^{* 0.059} \mathrm{e}^{-96.775 w_{\mathrm{s}}^{*}}
$$

This formula is valid for loads of up to $2000 \mathrm{~N}$, a speed of entraining motion down to a few $\mathrm{mm} / \mathrm{s}$, and squeeze-roll ratios of the order of $1 / 1000$ in mutual approach of the bodies (a negative value indicating approach). It has been successfully used for contact of wavy rotating surfaced discs [10] (which induces combined entraining and squeeze motion of the lubricant) in conformance with the experimental observations of Dareing and Johnson [11].

The dimensionless parameters in equation (7) are related to the instantaneous operating conditions, such as the contact load and rotational speed of the cam, lubricant rheology, and elastic constants of the materials in contact. These are given in the notation.

\subsection{Impact conditions in the valvetrain operation}

The elastodynamic model represented by the equation set (1) predicts the force acting on the cam-tappet contact. Loss of contact is represented by a zero contact load, which is followed by a subsequent rebounding of the tappet upon the cam. This impacting condition should be included in the model.

Figure 2 shows the forces applied on the camtappet contact during the engine operation for a rigid valvetrain model. Owing to the cam symmetry, only half of the cam-tappet operation cycle is shown. The elastic force is generated by the valve spring compression during the valve cycle. The inertial force is generated by the acceleration and mass of all the 


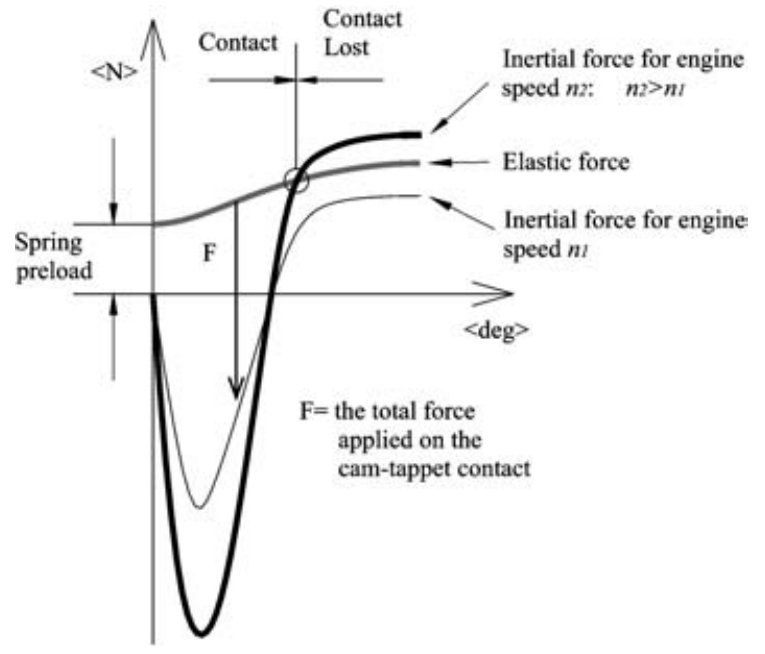

Fig. 2 Mechanism of loss of contact

valvetrain moving components. The elastic force is independent of the engine operating speed, and it is influenced only by the cam profile, valve spring stiffness, and valvetrain geometry. The inertial force depends on the speed of the moving components. In Fig. 2, the elastic force is shown, together with the inertial force, for two different engine speeds. The total force applied on the cam-tappet contact ( $F$ in Fig. 2) for each position represents the sum of elastic

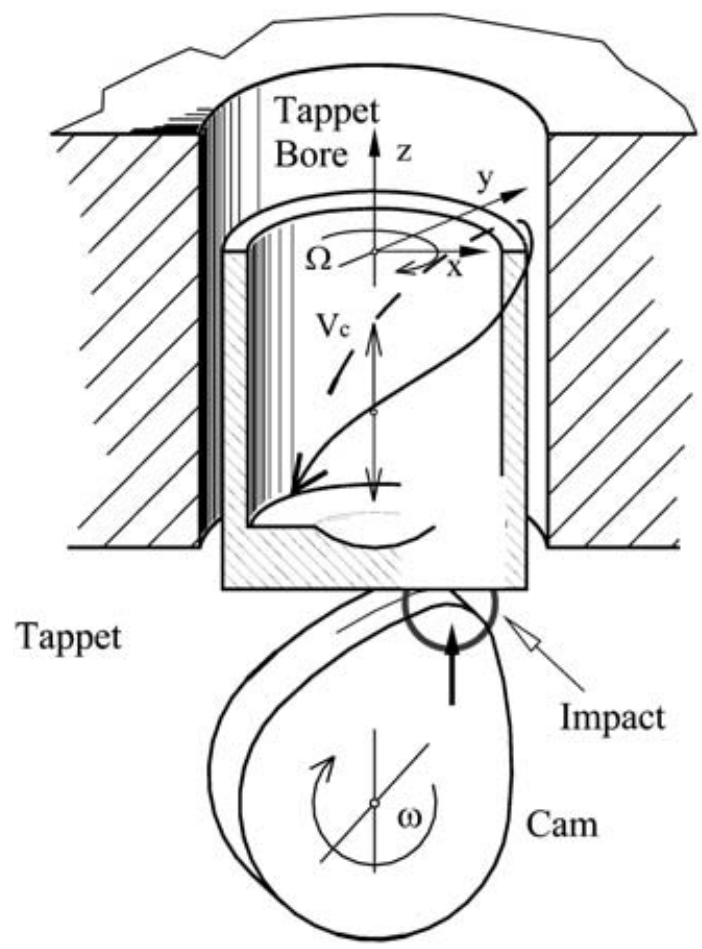

(a) Impact 1 and inertial forces. By convention, the force applied on the contact has a negative sign.

For a normal valvetrain operating condition, the elastic force is larger than the inertial force, and the cam-tappet contact is ensured. At location A, for the higher engine speed $n_{2}$, the inertial force becomes larger than the elastic force and the camtappet contact is lost. From this location on, the contact force becomes negative.

Two impact zones within the valvetrain system are illustrated in Fig. 3. Figure 3(a) shows the impact in the cam-tappet conjunction, which occurs with camshaft wind-up prior to cam nose and subsequent wind-down after it. The wind-up and wind-down are the combined result of camshaft elasticity and loading or loss of preload of the valvetrain components. Figure 3(b) shows the corresponding impact in the valve-valve seat contact. Both these impacts are highly undesirable, because they can lead to a number of untoward effects. These include loss of lubrication in the cam-tappet conjunction (contributing to frictional losses), wear of the valve seat, high inertial unbalance (contributing to powertrain mechanical losses), and potential structural damage.

The impact force is much larger than the usual contact force owing to the momentum-impulse transfer. To obtain a more realistic picture, and as

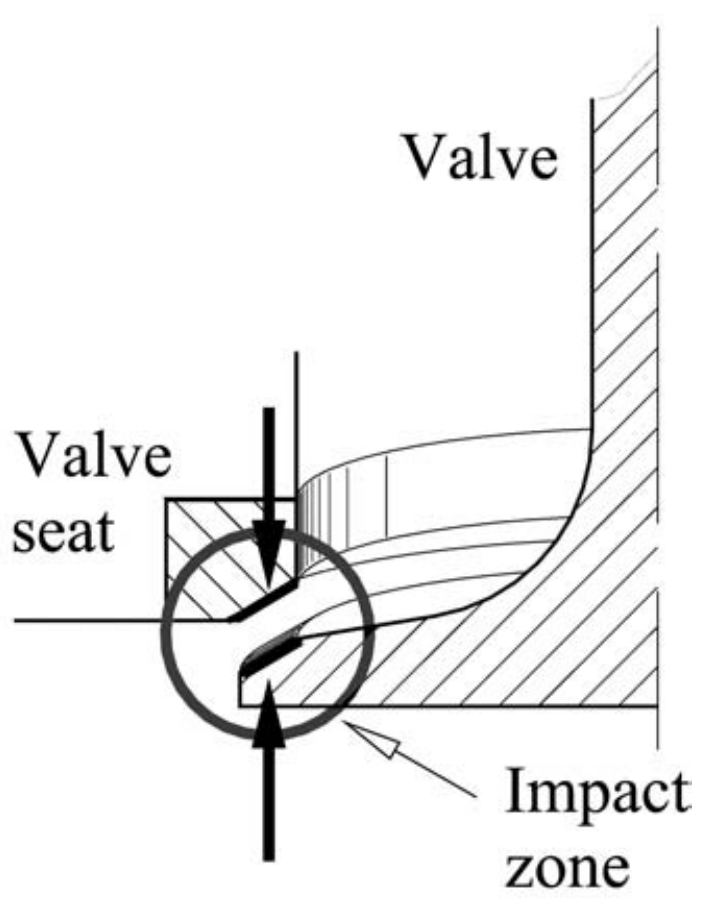

(b) Impact 2

Fig. 3 Significant impact zones in the valvetrain system 
an initial approximation, it may be assumed that the impact energy is insufficient to excite the structural modes of impacting members. This assumption is a restatement of localized nature of Hertzian impact theory. To use this approach, due consideration should be given to the geometric nature of the impacting solids, which in the case of a cam-tappet pair can be approximated by a roller of a radius equivalent to the instantaneous effective radius of the cam, impinging upon a semi-infinite elastic half-space plate. These conditions deviate from the classical Hertzian theory, but the limiting assumption of localized nature of energy exchange may be retained as valid.

The localized impact of two solids of revolution, $i=1,2$, can be described by the following equation

$$
m_{i} \frac{\mathrm{d} v_{i}}{\mathrm{~d} t}=m_{i} \dot{v}_{i}=-F, \quad i=1,2
$$

where the impact force $F$ opposes the direction of motion in the case of each solid, and by virtue of Newton's third axiom is the same for both the impacting bodies.

The velocity of approach is given by

$$
\dot{w}=\sum_{i=1}^{2} v_{i}
$$

Thus

$$
\ddot{w}=\sum_{i=1}^{2} \dot{v}_{i}=\dot{v}_{1}+\dot{v}_{2}=-F\left(\frac{m_{1}+m_{2}}{m_{1} m_{2}}\right)
$$

where $m_{\mathrm{eq}}=\left[\left(m_{1} m_{2}\right) /\left(m_{1}+m_{2}\right)\right]$ is the equivalent mass of a roller impacting a semi-infinite elastic half-space.

A relationship between the impact force and the mutual approach of the two impacting solids should be used for equation (10). For the case of finite line contact of a roller against an elastic half-space, Johns and Gohar [12] have shown that

$$
F=\frac{\pi L E^{\prime}}{2\left[\ln (2 L) / a+\frac{1}{2}\right]} w=K w
$$

where $a=\left[(8 F R) /\left(\pi E^{\prime} L\right)\right]^{1 / 2}$ and $p=(2 F) /(\pi a L)$, requiring a solution of these three equations in an iterative manner to obtain the impact force.

Substituting for the impact force in equation (10) and multiplying both sides of the equation by $\dot{w}$ yields

$$
\frac{1}{2} \frac{\mathrm{d} \dot{w}^{2}}{\mathrm{~d} t}=-\frac{K}{m_{\mathrm{eq}}} w \frac{\mathrm{d} w}{\mathrm{~d} t}
$$

Integrating both sides of the above equation

$$
\frac{1}{2}\left(\dot{w}^{2}-v^{2}\right)=-\frac{K}{2 m_{\mathrm{eq}}} w^{2}
$$

The maximum penetration is obtained when $\dot{w}=0$, which yields

$$
w_{\max }=\left(\frac{m v^{2}}{K}\right)^{1 / 2}=\left\{\frac{2\left[\ln (2 L) / a+\frac{1}{2}\right] m v^{2}}{\pi L E^{\prime}}\right\}^{1 / 2}
$$

Equation (10) can be rewritten as

$$
\left(\frac{\mathrm{d} w}{\mathrm{~d} t}\right)^{2}=v^{2}-\frac{K}{m_{\mathrm{eq}}} w^{2}
$$

Thus

$$
\mathrm{d} t=\left(v^{2}-\frac{K}{m_{\mathrm{eq}}} w^{2}\right)^{-1 / 2} \mathrm{~d} w
$$

Letting $x=w / w_{\max }$ and integrating the above equation yields the impact time as

$$
t_{\max }=\frac{2 w_{\max }}{v} \int_{0}^{1} \frac{1}{\sqrt{1-x^{2}}} \mathrm{~d} x=\frac{\pi w_{\max }}{v}
$$

The penetration in a Hertzian-type impact occurs in a symmetrical manner about the duration $1 / 2 t_{\max }$. Thus, the instantaneous penetration can also be found, using forward differences as

$$
w_{i}=w_{i-1}+\left[\operatorname{sign}\left(t_{\max }-2 \tau\right)\right] \dot{w}_{i-1} \Delta t, \quad 0 \leqslant \tau \leqslant t_{\max }
$$

where $\dot{w}_{i-1}$ is given by equation (13).

Therefore, equations (11), (13), and (18) describe the impact dynamics of the cam-tappet conjunction. Note that the lubricated nature of the impact is ignored in this approach. This assumption is reasonable, as the initial separation is beyond the limit that a lubricant film can be retained. Thus, in rebound of the tappet upon the cam, a coherent film does not exist. Nevertheless, the dry impacting condition can only be regarded as an approximation to the possible prevailing conditions.

\section{RESULTS AND DISCUSSION}

This paper investigates the tribological conditions, including the impact phenomenon in the cam-tappet conjunction. The impact phenomenon can be observed by monitoring valve acceleration. Figure 4(a) shows the accelerometer installed on the valve spring retainer of a single-cylinder Deutz F1L 210D diesel engine. The trace in the grey shade (Figure $4(\mathrm{~b})$ ) is the measured valve acceleration in a Deutz engine. 


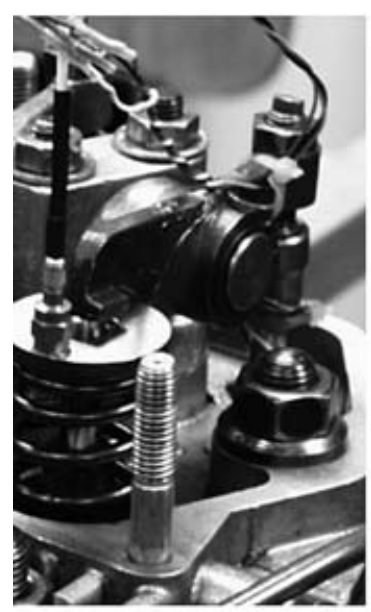

(a) Experiment

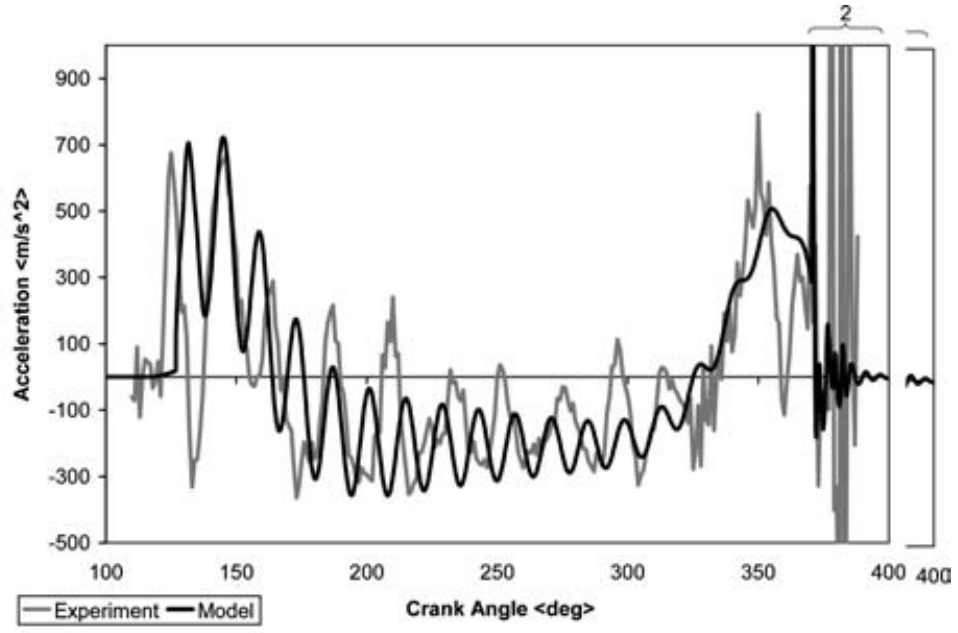

(b) Results

Fig. 4 Valve acceleration

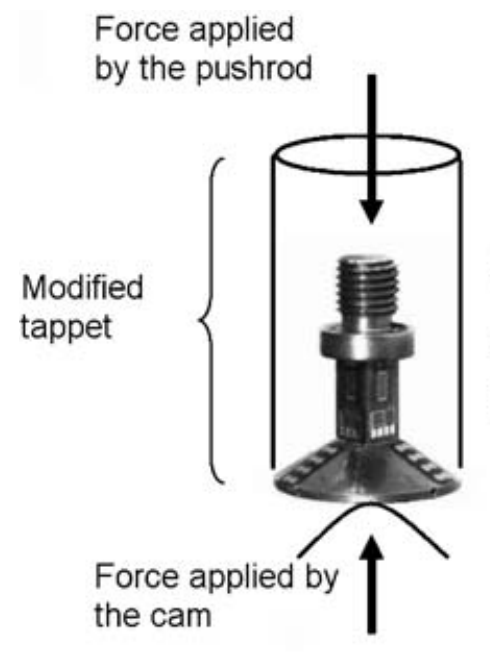

(a) Experiment

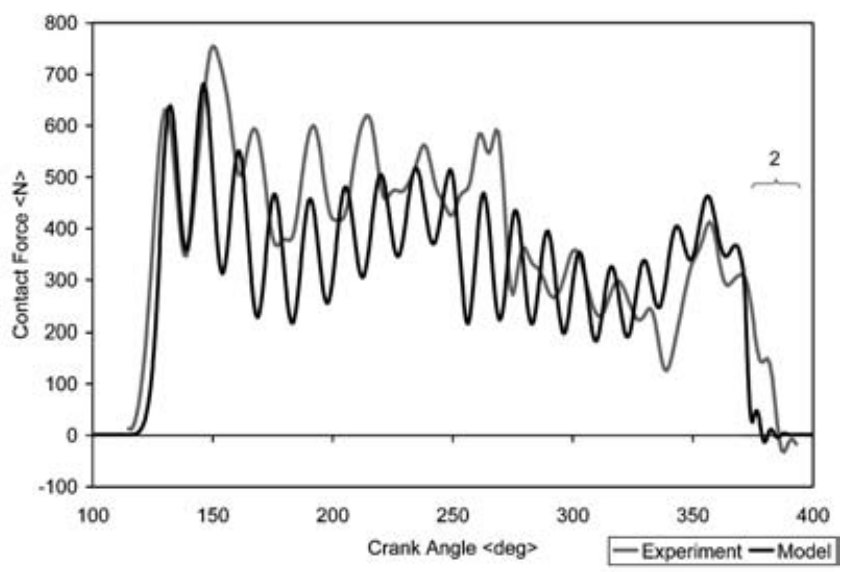

(b) Results

Fig. 5 Cam-tappet contact force

The cam nose location corresponds to the crank angle of $250^{\circ}$. Prior to this location, the event commences at the transition from the base circle to the cam flank, indicated by the geometrical acceleration, which leads to the cam nose contact. This event is associated with the wind-up process in which some strain energy is stored in the elastic camshaft, as well as in the valve spring and the other elastic members as illustrated in the model in Fig. 1. Associated with the wind-up process is some oscillatory behaviour of all the elastic members of the valvetrain system, as shown by the perturbation superimposed on the geometrical acceleration of the valve. The wind-down process is the combination of the release of this stored strain energy in the form of vibration of all the elastic members, culminating in large overshoot behaviour in transition back to the base circle. This is owing to loss of contact, because of the corresponding loss of preload, which is usually accompanied by a surge effect in the valve spring and flutter and repeated impacts in the valve-valve seat conjunction. Therefore, the behaviour of the two impact zones, shown in Fig. 3, is closely related. In Fig. 4(b), the trace in black is the predictions made by the model depicted in Fig. 1. Reasonable agreement is observed even with such a simplified model, indicating that for all practical purposes very detailed models are not necessary [4]. 
The corresponding contact force between the cam and the tappet reveals the nature of the aforementioned impacts. Note that a finite contact force is obtained both numerically and experimentally in reasonable accord for nearly all the cam cycle, except at the culmination of the wind-down. At this location the loss of contact occurs in the cam-tappet conjunction, indicated by a zero contact force. The impact occurs at the same time in the valve-valve seat contact, indicated in the figure by the digit ' 2 ' referring to this impact zone, as also indicated in Fig. 3. The oscillatory behaviour of the contact force, often misunderstood as repetitive impacts, is in fact merely owing to the cumulative effect of system compliance. This kind of analysis, in more detail, can be used to establish the limiting elasticity of the system, acceptable in striving to use materials of lighter construction, in order to reduce the translational imbalance in valvetrain systems.

The interactions between the loss of contact in one of the impact zones and the corresponding impact in the other may best be observed when an engine is run beyond its designed operating range. In the case of the Deutz engine, the useful operating range provides the maximum engine speed of about $2800 \mathrm{r} / \mathrm{min}$. Therefore, the valvetrain model was simulated at the engine speed of $3000 \mathrm{r} / \mathrm{min}$. One would expect the untoward phenomena of repetitive losses of contact and subsequent impacts in both the aforementioned impact zones, accompanied by a valve spring surge effect, as the system is simulated beyond its intended application speed (see Fig. 6). Figure 7 shows the oil-film thickness for the entire valve event (black line), as well as the instances of contact separation between the cam and the tappet (grey line). Each loss of contact is accompanied by subsequent impacts, visible in Fig. 6 as highfrequency, high-amplitude oscillations superimposed on the expected tappet acceleration. Note that, in the case of the engine speed of $3000 \mathrm{r} / \mathrm{min}$, there are successive losses of contact. During each camtappet impact, part of the energy required to deform the interacting bodies in the first half of the impact is dissipated, and consequently, even if in the immediate proximity of the impact the acceleration and the resultant contact force have a very high frequency and amplitude, in the long run, the impact will have a damping effect. This behaviour may be seen in Fig. 6(b) for the tappet acceleration and in Fig. 8(a) for cam-tappet contact force. In both situations, the grey line represents the behaviour neglecting the impact, and the black line represents the behaviour including the proposed impact model.

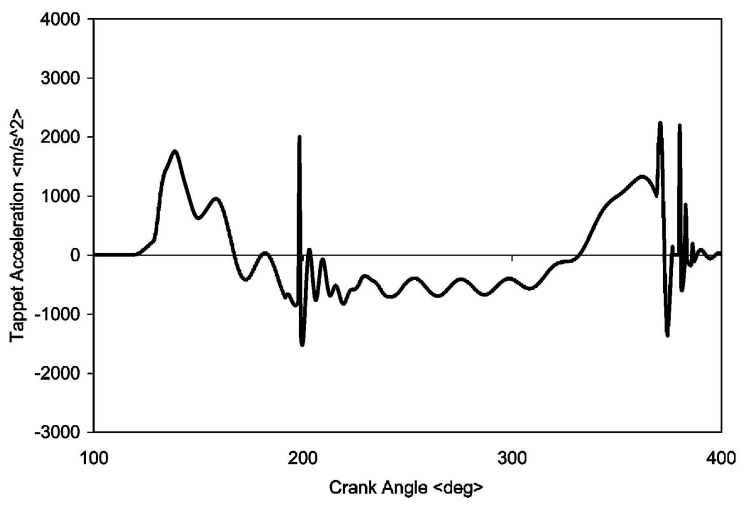

(a) $2800 \mathrm{rpm}$

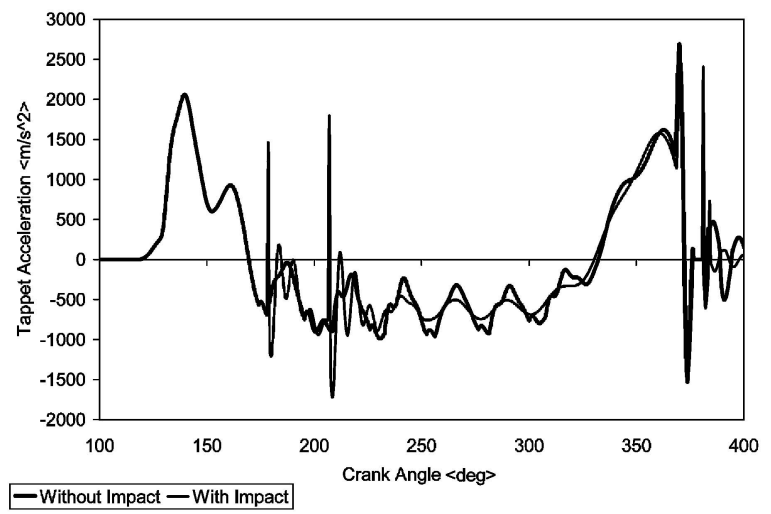

(b) $3100 \mathrm{rpm}$

Fig. 6 Tappet acceleration

Loss of contact will lead to adverse tribological conditions, chiefly the depletion of the lubricant film, as the gap becomes large enough with no or insufficient load to sustain a lubricant film. It is clear from Figs 6 and 7 that during the wind-up process the conditions are worse in the cam-tappet conjunction, whereas the problem is mostly transferred to the valve-valve seat contact during the wind-down event. The contact load variation for the engine speed of $3000 \mathrm{r} / \mathrm{min}$ is shown in Fig. 8(a). Note that loss of contact may be observed where the contact force diminishes. What is not taken into account in the generation of the time history of the contact force in Fig. 8(a) is the impact force generated by the rebound of the valve upon the rotating cam. This occurs as a result of a preceding impact in the valvevalve seat contact, transmitted through the system, which itself has been caused by a previous instance of the separation effect in the cam-tappet conjunction. This, of course, is a manifestation of Newton's third axiom, although some attenuation of this toand-fro action takes place owing to the structural damping of the system elements, and in particular to frictional losses in the rocker arm bearing.

The importance of understanding the mechanisms that lead to valve spring surge and loss of contact in 


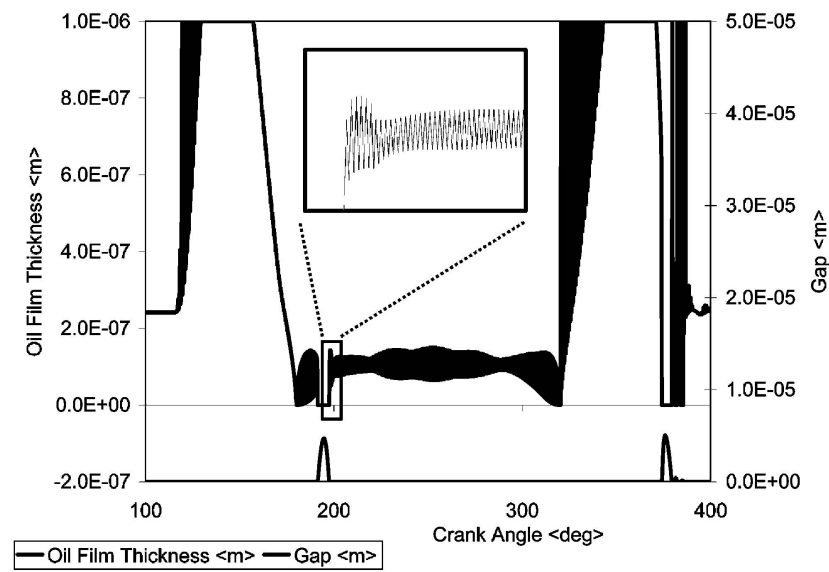

(a) $2800 \mathrm{rpm}$

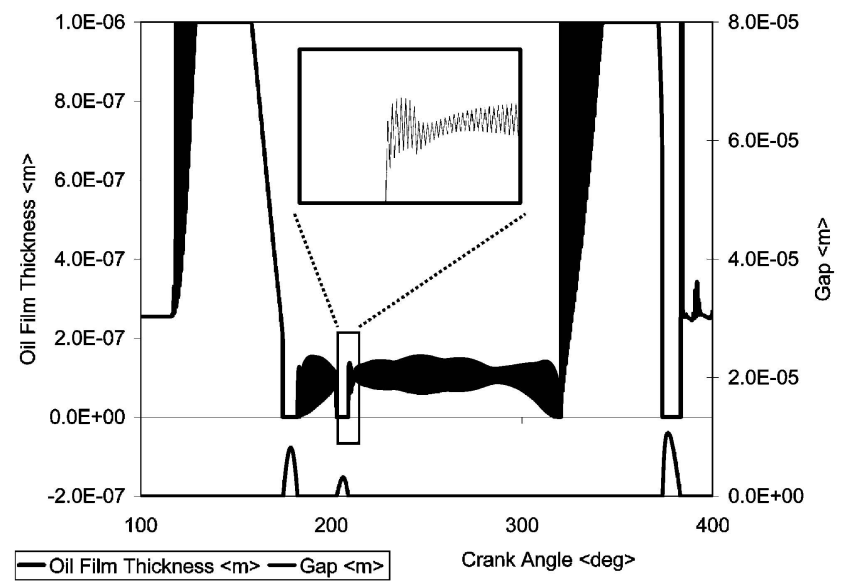

(b) $3000 \mathrm{rpm}$

Fig. 7 Oil-film thickness

the cam-tappet conjunction is emphasized by the fact that, irrespective of engine speed, the first impact always occurs in very proximate location to the cam-tappet inlet speed reversal point (see Figs 6, 7, and $8(\mathrm{~b})$ ). The cyclic nature of this impact can lead to scarring of the cam surface at these locations, either by wear (owing to insufficient lubrication) or by pitting (owing to high subsurface stresses. At this location the entraining velocity is zero and the only mechanism responsible for maintaining the oil film is surmised to be the squeeze-film effect (see equation (7)). The oil-film thickness is at its minimum and the possibility of surface asperity contact (between the approaching bodies) is greatly increased. If, however, this location coincides with the cam-tappet impact, the tribological conditions deteriorate even further, with possible adverse consequences. The diminishing oil-film thickness, followed by the surface asperity interaction, is the

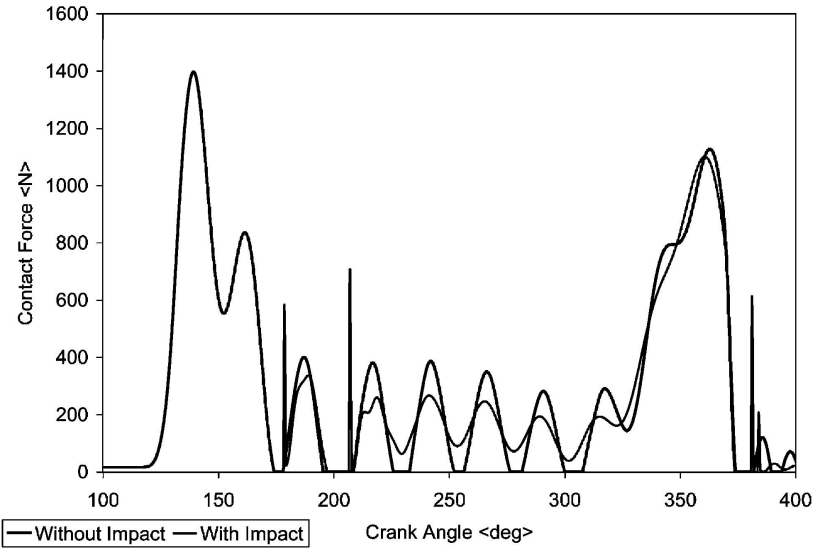

(a) Contact Force

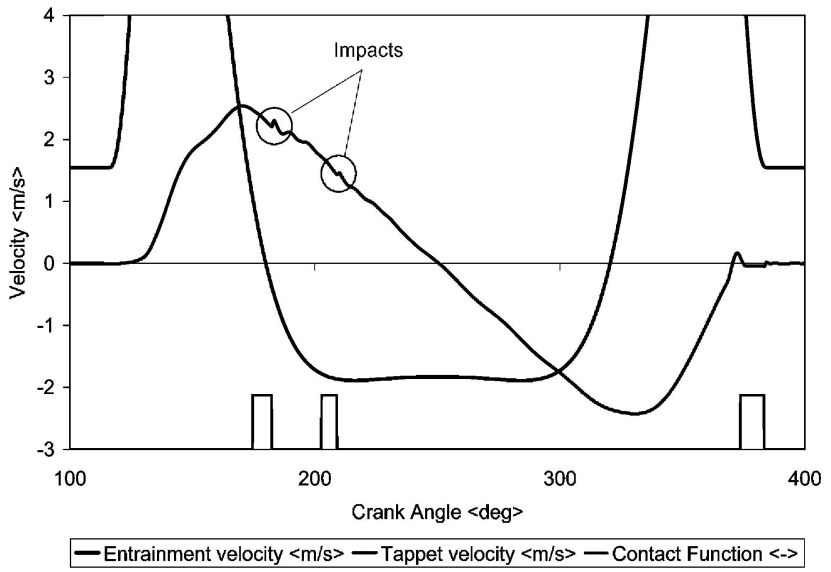

(b) Contact conditions for cam-tappet conjunction

Fig. 8 Contact conditions at $3000 \mathrm{r} / \mathrm{min}$

major factor responsible for the boundary friction force in the cam-tappet contact and consequently the wear of the two sliding surfaces.

\section{CONCLUSIONS}

The paper shows that a relatively simple two-mass model of the valvetrain system, incorporating all aspects of interacting phenomena, referred to here as a multiphysics approach, can yield predictions that conform to experimental findings, as well as providing a fundamental understanding of these complex interactions. It is also shown that the trend set in the use of materials of lighter construction, without regard to other interacting phenomena, is ill-conceived in terms of aggravating other adverse tribological and non-linear impact conditions. 


\section{ACKNOWLEDGEMENTS}

The authors would like to express their gratitude to the SKF Engineering Research Centre in Nieuwegein, the Netherlands, for the financial support extended to this project, as well as the support of the US Army National Automotive Center, and TARDEC, Warren, MI.

\section{REFERENCES}

1 Kushwaha, M. and Rahnejat, H. Transient elastohydrodynamic lubrication of finite line conjunction of cam to follower concentrated contact, J. Phys. D: Appl. Phys., 2002, 35(3), 2872-2890.

2 Kushwaha, M., Rahnejat, H., and Jin, Z. M. Valvetrain dynamics: a simplified tribo-elasto-multi-body analysis, Proc. IMechE, Part K: J. Multi-Body Dynamics, 2000, 214(K2), 95-110.

3 Koster, M. P. Effect of flexibility of driving shaft on the dynamic behaviour of a cam mechanism, Trans. ASME, J. Engng for Ind., 1975, 595-602.

4 Teodorescu, M., Kushwaha, M., Rahnejat, H., and Taraza, D. Elastodynamic transient analysis of a four-cylinder valvetrain system with camshaft flexibility, Proc. IMechE, Part K: J. Multi-Body Dynamics, 2005, 219(K1), 13-25.

5 Teodorescu, M. and Taraza, D. Numerical prediction and experimental investigation of cam to flat tappet lubricated contact with tappet spin, Proc. IMechE, Part K: J. Multi-Body Dynamics, 2004, 218(K3), 133-142.

6 Teodorescu, M. Modular approach for valve train dynamics and friction simulation with in situ experimental validation, $\mathrm{PhD}$ thesis, Wayne State University, Detroit, MI, 2003.

7 Chen Fan, Y. Mechanics and Design of Cam Mechanisms, 1982 (Pergamon Press).

8 Jalali-Vahid, D., Kushwaha, M., and Rahnejat, $\mathbf{H}$. Transient concentrated lubricated contact dynamics, Int. J. Gearing and Transm., 2000, 3, 76-82.

9 Rahnejat, H. Influence of vibration on the oil film in concentrated contacts, $\mathrm{PhD}$ thesis, Imperial College of Science and Technology, London, 1984.

10 Mehdigoli, H., Rahnejat, H., and Gohar, R. Vibration response of wavy surfaced disc in elastohydrodynamics rolling contact, Wear, 1990, 139, $1-15$.

11 Dareing, D. W. and Johnson, K. L. Fluid film damping of rolling contact vibrations, Proc. IMechE, Part C: J. Mechanical Engineering Science, 1975, 17, 214-218.

12 Johns, P. M. and Gohar, R. Roller bearing under radial and eccentric loads, Tribology Int., 1981, pp. 131-136.

\section{APPENDIX}

\section{Notation}

$a \quad$ contact width (m)

$c_{\mathrm{e}} \quad$ equivalent damping coefficient $(\mathrm{N} \mathrm{s} / \mathrm{m})$

$c_{i} \quad$ generic damping coefficient $(\mathrm{N} \mathrm{s} / \mathrm{m})$

$C_{i} \quad$ polynomial coefficients

$E_{1}, E_{2} \quad$ elastic moduli for cam and follower materials $(\mathrm{Pa})$

$E^{\prime}$

$\frac{1}{E^{\prime}}=\frac{1}{2}\left(\frac{1-v_{1}^{2}}{E_{1}}+\frac{1-v_{2}^{2}}{E_{2}}\right)$

$f_{i} \quad$ friction force associated with mass $i(\mathrm{~N})$

$F \quad$ impact force (N)

$F^{*} \quad$ non-dimensional load $=W /\left(E^{\prime} R_{\mathrm{c}} L\right)$

$F_{i}^{m} \quad$ external force applied on equivalent mass $i(\mathrm{~N})$

$G^{*} \quad$ non-dimensional material parameter $=\alpha E^{\prime}$

$h_{\mathrm{o}}^{*} \quad$ central oil-film thickness (m)

$k_{\mathrm{e}} \quad$ equivalent stiffness $(\mathrm{N} / \mathrm{m})$

$k_{i} \quad$ generic stiffness $(\mathrm{N} / \mathrm{m})$

$K \quad$ contact stiffness $(\mathrm{N} / \mathrm{m})$

$l_{i}$

generic mass movement at time step $i$

(m)

$L \quad$ cam (generic roller) width (m)

$m \quad$ equivalent impacting mass (m)

$m_{1} \quad$ pushrod and tappet equivalent mass $(\mathrm{kg})$

$m_{2}$

$m_{\text {eq }}$

$p$

$r_{i}$

$R_{\mathrm{c}}$

$t, \tau$

$t_{\max }$

$u$

$u_{i}$

$U^{*}$

$v_{i}$

$w$

valve assembly equivalent mass $(\mathrm{kg})$

equivalent mass $(\mathrm{kg})$

contact pressure $(\mathrm{Pa})$

polynomial powers

curvature radius $(\mathrm{m})$

time coordinate (s)

impact time (s)

speed of lubricant entrainment $(\mathrm{m} / \mathrm{s})$

velocity of surface $i(\mathrm{~m} / \mathrm{s})$

non-dimensional speed $=\left(\eta_{0} u\right) /\left(E^{\prime} R_{\mathrm{c}}\right)$

impact velocity of body $i(\mathrm{~m} / \mathrm{s})$

mutual approach of contacting solids (m)

maximum penetration (m)

$\begin{array}{ll}w_{\max } & \text { squeeze-roll ratio } \\ w_{\mathrm{s}}^{*} & \end{array}$

$x \quad$ floor movement (m)

$y$

$z$

mass $m_{1}$ movement $(\mathrm{m})$

mass $m_{2}$ movement $(\mathrm{m})$

$\alpha$

piezo-viscosity index $\left(\mathrm{Pa}^{-1}\right)$

residual error term $(\mathrm{m})$

$\varepsilon_{\text {res }}$

$v_{1,2}$

$\omega$
Poisson's ratios for cam and follower materials

camshaft angular velocity ( $\mathrm{rad} / \mathrm{s}$ ) 